\title{
Dynamic Control of Soft-finger Hands for Pivoting an Object in Contact with the Environment
}

\author{
T. Yoshikawa and T. Watanabe \\ Department of Mechanical Engineering \\ Kyoto University \\ Kyoto, 606-8501 Japan \\ \{yoshi@mech.,t60x0113@ip.media.\}kyoto-u.ac.jp
}

\begin{abstract}
In this paper, we propose a dynamic control method of soft-finger hands for pivoting an object in contact with the environment. This pivoting operation is often observed when a human moves a large or heavy object such as furniture. Different from the conventional manipulation of the object by only fingers, the characteristics of the pivoting operation is that we can use the reaction force from the environment. By using this reaction force, we can expect the magnitude of the forces applied to the object by the fingers is smaller than the conventional manipulation of the object by only fingers. In this paper, taking this characteristics of the reaction force into consideration, we propose a dynamic control method for pivoting. To verify our approach, the simulation result is also presented.
\end{abstract}

\section{Introduction}

Many researchers have studied the manipulation of an object which contacts with an environment such as a table. While the environment constrains the motion of the object, we can use the reaction force from the environment in the manipulation of the object. Then, we can expect that the needed number of the fingers to manipulate the object is smaller than the number of fingers needed to manipulate the object by only fingers such as Li et al.[1], Cole et al.[2], and Yokokouji et al.[3]. In this paper, the goal is to establish a control method for forming the force closure[4] grasp and manipulating the object in contact with the environment by a few fingers even when we cannot form the force closure grasp by only fingers. Especially, we are interested in pivoting operation. Pivoting operation is a rotation of the object around an axis through a contact point between the object and the environment. This manipulation is often observed when a human moves a large or heavy object such as furniture. Aiyama et al[5] have proposed the quasi-static motion planning for manipulating a rectangular object, but we cannot apply the planning to a general object. On the other hand, some manipulations similar to the pivoting have been studied. Han et al.[7] proposed a control method for the quasi-static manipulation of the spherical object on a plane by one finger which has
6 D.O.F. and a flat fingertip. Trinkle et al.[8] quasistatically analyzed the manipulation of lifting up the object on the support. In another paper[9], Trinkle et al. proposed a quasi-static manipulation planning for enveloping the slippery work piece on the palm and manipulating it. But the dynamic control method for these manipulations has been studied only by a few researchers (for example, Yoshikawa et al.[6], but, the control method is proposed under the assumption that we can form the force closure grasp by only fingers ). In this paper, we propose a dynamic control method of soft-finger hands for pivoting an object in contact with the environment.

This paper is organized as follows. In section 2, we describe the target system of this paper for pivoting, and in section 3, we develop a dynamic control method for pivoting. First, we formulate the kinematic constraint between the object and the fingers and constraint between the object and the environment. Second, we formulate the dynamics of the object and the fingers. Third, we formulate the contact forces and moment. Forth, we formulate the internal forces and derive the magnitude of the components of the internal forces, which minimizes the force applied to the object by the fingers by using the nonlinear programming. Based on the formulations, we develop the controller for pivoting. To verify our approach, we show a simulation result in section 4 .

\section{Target System}

The target system of this paper is shown in Fig.1. This system consists of two fingers and an object. each finger has three joints and a spherical soft fingertip, and the object contacts the environment at one vertex. The given task is to pivot the object in contact with the environment at the vertex by the two fingers along with a desired trajectory. We make the following assumption; $(i)$ the object is rigid and its contact surface is smooth, and (ii) the object contacts with the environment at the fixed point. For the analysis of the soft fingertips, we also assume that contact area is so small that the contact between the soft fingertip and the object is regarded as a point contact, that tangential frictional force and frictional twist/spin moment 
around the contact normal are independent and they are approximated by the Coulomb model, and that energy dissipation due to the fingertip deformation is negligible.

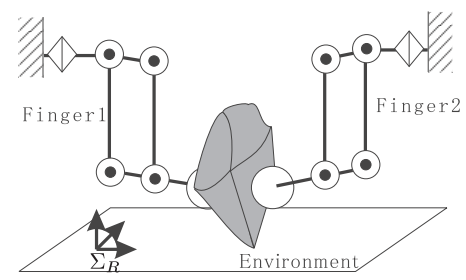

Figure 1: Target System

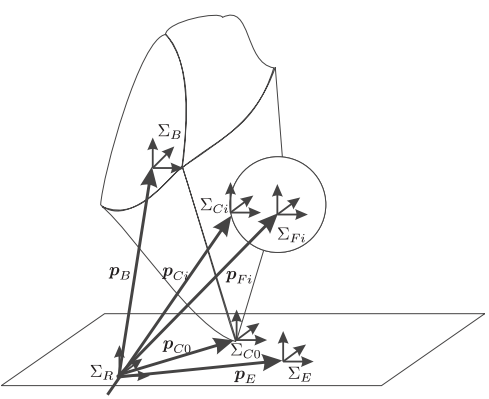

Figure 2: Objet, fingers, and Environment

\section{Control Scheme}

In this section, we propose the control scheme based on the formulations by Li et al.[1], Cole et al.[2], Yokokouji et al.[3], and Yoshikawa et al.[6]. Note that we permit a rotational slide around the contact normal at the contact point between the finger and the object.

\subsection{Kinematics Constraints}

In this subsection, we formulate the kinematic constraint between the fingers and the object and constraint between the environment and the object. As shown in Fig. $2, \Sigma_{R}, \Sigma_{B}, \Sigma_{F i}$, and $\Sigma_{C_{i}}(i=0,1,2)$ denote a reference coordinate frame, a object coordinate frame placed at the gravity center, a fingertip coordinate frame placed at the center of the spherical fingertip of the finger $i,(i=1,2)$, and a frame fixed on the object surface at the contact point $C_{i}$, respectively. Let the position of the origin and the orientation of $\Sigma_{a}$ with respect to $\Sigma_{c}\left(\Sigma_{R}\right)$ be given by ${ }^{c} \boldsymbol{p}_{a}\left(\boldsymbol{p}_{a}\right)$ and ${ }^{c} \boldsymbol{R}_{a}\left(\boldsymbol{R}_{a}\right)$, respectively. Let a vector directing from the origin of $\Sigma_{a}$ to the origin of $\Sigma_{b}$ with respect to $\Sigma_{c}$ $\left(\Sigma_{R}\right)$ be given by ${ }^{c} \boldsymbol{p}_{a b}\left(\boldsymbol{p}_{a b}\right)$.

First, the constraint at the fixed contact point between the environment and the object is given by

$$
\boldsymbol{H}_{C 0} \boldsymbol{D}_{B 0}\left(\begin{array}{c}
\dot{\boldsymbol{p}}_{B} \\
\boldsymbol{\omega}_{B}
\end{array}\right)=\boldsymbol{H}_{C 0}\left(\begin{array}{c}
\dot{\boldsymbol{p}}_{C 0} \\
\boldsymbol{\omega}_{C 0}
\end{array}\right)=\dot{\boldsymbol{p}}_{C 0}=\mathbf{o}
$$

where

$$
\begin{aligned}
\boldsymbol{D}_{B i} & =\left(\begin{array}{cc}
\boldsymbol{I}_{3} & -\left[\left(\boldsymbol{R}_{B}^{B} \boldsymbol{p}_{B C i}\right) \times\right] \\
\boldsymbol{O}_{3} & \boldsymbol{I}_{3}
\end{array}\right) \\
\boldsymbol{H}_{C 0} & =\left(\begin{array}{ll}
\boldsymbol{I}_{3} & \boldsymbol{O}_{3}
\end{array}\right)
\end{aligned}
$$

Here, $\boldsymbol{\omega}_{B}$ and $\boldsymbol{\omega}_{C i}$ denote an angular velocities of the object $\left(\Sigma_{B}\right)$ and $\Sigma_{C i},[\times]$ denotes a skew symmetric equivalent to the cross product operation, and $\boldsymbol{I}_{*}$ and $\boldsymbol{O}_{*}$ denote the $*$-order identity and null matrices, respectively. Here, instead of the angular velocity of the object $\boldsymbol{\omega}_{B}$, we use roll, pitch and yaw angles for expressing the object orientation. The relation between $\boldsymbol{\omega}_{B}\left(=\boldsymbol{\omega}_{C 0}\right)$ and the velocity of roll, pitch and yaw angles, $\dot{\boldsymbol{\phi}}_{B}\left(=\dot{\boldsymbol{\phi}}_{C 0}\right)$, are given by $\boldsymbol{\omega}_{B}=\boldsymbol{T}_{r} \dot{\boldsymbol{\phi}}_{B}$ where $\boldsymbol{T}_{r}\left(\phi_{B}\right)$ denotes the matrix for the transformation. Then, from (1), we get

$$
\boldsymbol{D}_{B 0}\left(\begin{array}{c}
\dot{\boldsymbol{p}}_{B} \\
\boldsymbol{\omega}_{B}
\end{array}\right)=\left(\begin{array}{c}
\dot{\boldsymbol{p}}_{C 0} \\
\boldsymbol{\omega}_{C 0}
\end{array}\right)=\boldsymbol{T}\left(\begin{array}{c}
\dot{\mathbf{o}}_{C 0} \\
\dot{\boldsymbol{\phi}}_{C 0}
\end{array}\right)
$$

where $\boldsymbol{T}=\operatorname{diag}\left[\boldsymbol{I}_{3} \boldsymbol{T}_{r}\left(\boldsymbol{\phi}_{C 0}\right)\right]$ ("diag" means a block diagonal matrix).

Second, since the contact between the finger and the object is a soft-finger type contact, the constraint at the contact point between the finger $i(i=1,2)$ and the object is given by

$\boldsymbol{H}_{C i} \boldsymbol{D}_{B i}\left(\begin{array}{c}\dot{\boldsymbol{p}}_{B} \\ \boldsymbol{\omega}_{B}\end{array}\right)=\boldsymbol{H}_{C i} \boldsymbol{D}_{F i}\left(\begin{array}{c}\dot{\boldsymbol{p}}_{F i} \\ \boldsymbol{\omega}_{F i}\end{array}\right)=\boldsymbol{H}_{C i}\left(\begin{array}{c}\boldsymbol{v}_{C i} \\ \boldsymbol{\omega}_{C i}\end{array}\right)$

where

$$
\begin{aligned}
& \boldsymbol{D}_{F i}=\left(\begin{array}{cc}
\boldsymbol{I}_{3} & -\left[\left(\boldsymbol{R}_{F i}{ }^{F i} \boldsymbol{p}_{F i C i}\right) \times\right] \\
\boldsymbol{O}_{3} & \boldsymbol{I}_{3}
\end{array}\right) \\
& \boldsymbol{H}_{C i}= \begin{cases}\left(\begin{array}{cc}
\boldsymbol{I}_{3} & \boldsymbol{O}_{3} \\
\mathbf{o} & \boldsymbol{n}_{C i}^{T}
\end{array}\right) & \text { (not spinning) } \\
\left(\begin{array}{ll}
\boldsymbol{I}_{3} & \mathbf{o}
\end{array}\right) & \text { (spinning) }\end{cases}
\end{aligned}
$$

Here, $\boldsymbol{v}_{C i}$ denotes a vector of the contact point velocity, where the components of the contact point movement due to the rolling are excluded, and $\boldsymbol{\omega}_{F i}$ denotes an angular velocities of the finger $i\left(\Sigma_{F i}\right)$.

Finally, the relation between the fingertip velocity of the finger $i$ and the joint velocities of the finger $i$ is given by

$$
\left(\begin{array}{c}
\dot{\boldsymbol{p}}_{F i} \\
\boldsymbol{\omega}_{F i}
\end{array}\right)=\boldsymbol{J}_{F i} \dot{\boldsymbol{q}}_{i}
$$

where $\boldsymbol{J}_{F i}$ denotes a Jacobian matrix of the finger $i$ and $\boldsymbol{q}_{i}$ denotes a joint vector of the finger $i$.

From (4) (5) (8), we get

$$
\begin{aligned}
& \boldsymbol{H}_{C i} \boldsymbol{D}_{B i} \boldsymbol{D}_{B 0}^{-1} \boldsymbol{T}\left(\begin{array}{c}
\mathbf{o} \\
\dot{\boldsymbol{\phi}}_{C 0}
\end{array}\right)=\boldsymbol{H}_{C i}\left(\begin{array}{c}
\boldsymbol{v}_{C i} \\
\boldsymbol{\omega}_{C i}
\end{array}\right) \\
= & \boldsymbol{H}_{C i} \boldsymbol{D}_{F i} \boldsymbol{J}_{F i} \dot{\boldsymbol{q}}_{i}=\boldsymbol{J}_{C F M i} \dot{\boldsymbol{q}}_{i}
\end{aligned}
$$

\subsection{Dynamics of Fingers and Object}

In this subsection, we formulate the dynamics of the fingers and the object. Let $\boldsymbol{t}_{B}$ be the total forces and moments applied to the object by the fingers at the origin of the frame $\Sigma_{B}$, and $\boldsymbol{f}_{f}$ be the force applied to the environment by the object at the contact point (note that $-\boldsymbol{f}_{f}$ expresses the reaction force). Then, from 
(1), the resultant force be given by $\boldsymbol{t}_{B}-\boldsymbol{D}_{B 0}^{T} \boldsymbol{H}_{C 0}^{T} \boldsymbol{f}_{f}$. So, the dynamics of the object is given by

$$
\boldsymbol{t}_{B}-\boldsymbol{D}_{B 0}^{T} \boldsymbol{H}_{C 0}^{T} \boldsymbol{f}_{f}=\boldsymbol{M}_{B}\left(\begin{array}{c}
\ddot{\boldsymbol{p}}_{B} \\
\dot{\boldsymbol{\omega}}_{B}
\end{array}\right)+\boldsymbol{h}_{B}=\hat{\boldsymbol{t}_{B}}
$$

where $\boldsymbol{M}_{B}$ denotes an inertia tensor of the object and $\boldsymbol{h}_{B}$ denotes centrifugal, Coriolis and gravitational forces.

Next, the dynamics of the finger $i$ is given by

$$
\boldsymbol{M}_{F}(\boldsymbol{q}) \ddot{\boldsymbol{q}}+\boldsymbol{h}_{F}(\boldsymbol{q}, \dot{\boldsymbol{q}})=\boldsymbol{\tau}-\boldsymbol{J}^{T} \boldsymbol{f}_{S}
$$

where $\tau=\operatorname{col}\left[\tau_{1}, \tau_{2}\right] \in R^{6}$ ("col" means a column vector or matrix formed by the following matrix) $\left(\boldsymbol{\tau}_{i} \in R^{3}\right.$ denotes a joint torque of the finger $i), \boldsymbol{f}_{S}=\operatorname{col}\left[\boldsymbol{f}_{S 1}, \boldsymbol{f}_{S 2}\right]$ ( $\boldsymbol{f}_{S i}$ denotes the force and the moment applied to the object by finger $i$ at the contact point $C_{i}$ expressed by a vector representing the magnitudes of the components in the directions expressed by a column vector of $\boldsymbol{H}_{C i}$ in (7)), $\boldsymbol{M}_{F}=\operatorname{diag}\left[\boldsymbol{M}_{F 1}\right.$ $\left.\boldsymbol{M}_{F 2}\right]\left(\boldsymbol{M}_{F i}\right.$ denotes a inertia matrix of the finger $\left.i\right)$, $\boldsymbol{h}_{F}=\operatorname{diag}\left[\boldsymbol{h}_{F 1} \boldsymbol{h}_{F 2}\right]\left(\boldsymbol{h}_{F i}\right.$ denotes centrifugal, Coriolis and gravitational forces), and $\boldsymbol{J}=\boldsymbol{J}_{C F M}+\boldsymbol{J}_{s m}$ where $\boldsymbol{J}_{C F M}=\operatorname{diag}\left[\boldsymbol{J}_{C F M 1} \boldsymbol{J}_{C F M 2}\right]($ equation(9)), and $\boldsymbol{J}_{s m}=\operatorname{diag}\left[\begin{array}{ll}\boldsymbol{J}_{s m 1} & \boldsymbol{J}_{s m 2}\end{array}\right]$. Here, $\boldsymbol{J}_{s m i}$ is represented by

$$
\begin{array}{r}
\boldsymbol{J}_{s m i}^{T}=\boldsymbol{J}_{F i}^{T}\left(\begin{array}{ll}
\boldsymbol{O}_{3} & \boldsymbol{O}_{3} \\
\boldsymbol{N}_{i} & \boldsymbol{O}_{3}
\end{array}\right) \boldsymbol{H}_{C i}^{T} \\
\boldsymbol{N}_{i}=\alpha_{i} \mu_{i} \boldsymbol{n}_{C i} \boldsymbol{n}_{C i}^{T}
\end{array}
$$

where $\boldsymbol{n}_{C i}$ denotes the contact normal, $\alpha_{i}(=-1,0$, or 1$)$ shows the direction of the frictional twist/spin moment at the contact point $C_{i}$, and $\mu_{i}$ denotes the dynamic rotational frictional coefficient of the finger $i$. Note that $\boldsymbol{J}_{s m i} \boldsymbol{f}_{S i}$ expresses a joint torque equivalent to the dynamic frictional twist/spin moment, $\left[\begin{array}{ll}\boldsymbol{O}_{3} & \boldsymbol{N}_{i}\end{array}\right] \boldsymbol{H}_{C i}^{T} \boldsymbol{f}_{S i}$.

\subsection{Contact Forces and Moment}

In this subsection, we formulate the force and the moment applied to the object by the fingers at the contact points, $\boldsymbol{f}_{S}$, and the reaction force, $-\boldsymbol{f}_{f}$.

First, we formulate $\boldsymbol{f}_{S}$. From (5) (13) and the principle of virtual work, the relation between $\boldsymbol{t}_{B}$ and $\boldsymbol{f}_{S}$ is given by

$$
\begin{aligned}
\boldsymbol{t}_{B} & =\tilde{\boldsymbol{A}}\left(\boldsymbol{J} \boldsymbol{f}_{C}+\tilde{\boldsymbol{J}} \tilde{\boldsymbol{f}}_{C}\right)=\boldsymbol{A} \boldsymbol{f}_{C}+\tilde{\boldsymbol{A}} \tilde{\boldsymbol{J}} \tilde{\boldsymbol{f}}_{C} \\
\tilde{\boldsymbol{A}} & =\left(\boldsymbol{D}_{B}^{T}+\left(\begin{array}{llll}
\boldsymbol{O}_{3} & \boldsymbol{O}_{3} & \boldsymbol{O}_{3} & \boldsymbol{O}_{3} \\
\boldsymbol{N}_{1} & \boldsymbol{O}_{3} & \boldsymbol{N}_{2} & \boldsymbol{O}_{3}
\end{array}\right)\right) \boldsymbol{H}_{C}^{T}(15)
\end{aligned}
$$

where $\boldsymbol{D}_{B}=\operatorname{col}\left[\begin{array}{ll}\boldsymbol{D}_{B 1} & \boldsymbol{D}_{B 2}\end{array}\right], \boldsymbol{H}_{C}=\operatorname{diag}\left[\begin{array}{ll}\boldsymbol{H}_{C 1} & \boldsymbol{H}_{C 2}\end{array}\right]$, $\boldsymbol{f}_{S}=\boldsymbol{J} \boldsymbol{f}_{C}+\tilde{\boldsymbol{J}} \tilde{\boldsymbol{f}}_{C}$, and $\tilde{\boldsymbol{J}}_{\text {is }}$ an arbitrary matrix which satisfies $\operatorname{rank}[\boldsymbol{J} \tilde{\boldsymbol{J}}]=8$. Note that $\boldsymbol{J} \boldsymbol{f}_{C}$ expresses the term which can be applied to the object by joint torques, and $\tilde{\boldsymbol{J}} \tilde{\boldsymbol{f}}_{C}$ is expresses the term which cannot be applied. From (14), we get

$$
\boldsymbol{f}_{C}=\boldsymbol{A}^{+}\left(\boldsymbol{t}_{B}-\tilde{\boldsymbol{A}} \tilde{\boldsymbol{J}} \tilde{\boldsymbol{f}}_{C}\right)+\boldsymbol{\Phi} \tilde{\boldsymbol{f}}
$$

where $\boldsymbol{\Phi}$ is an arbitrary matrix which satisfies $\boldsymbol{A} \boldsymbol{\Phi}=\mathbf{o}$ , $\tilde{\boldsymbol{f}}$ express the magnitude of the component in the direction expressed by a column vector of $\boldsymbol{\Phi}$, and .*+ denotes the pseudo-inverse matrix of $*$.

Next, we formulate $\boldsymbol{f}_{f}$. There exist two subjects in this formulation. One is to formulate $\boldsymbol{f}_{f}$ which complements the dynamics of the object with $\boldsymbol{f}_{S}$, namely, $\boldsymbol{f}_{C}$, when $\boldsymbol{f}_{C}$ with $\boldsymbol{f}_{f}=\mathbf{o}$ cannot complements the dynamics absolutely. Two is to formulate $\boldsymbol{f}_{f}$ which minimizes the norm of the forces applied to the object by the fingers, $\boldsymbol{f}_{S}$. In order to achieve two subjects, we consider the following problem.

$$
\begin{aligned}
& \min \boldsymbol{f}_{S}^{T} \boldsymbol{f}_{S}=\boldsymbol{f}_{C}^{T} \boldsymbol{J}^{T} \boldsymbol{J} \boldsymbol{f}_{C} \\
& \text { subj. to. } \quad \mathbf{o}=\left(\boldsymbol{I}-\boldsymbol{A} \boldsymbol{A}^{+}\right)\left(\boldsymbol{D}_{B 0}^{T} \boldsymbol{H}_{C 0}^{T} \boldsymbol{f}_{f}+\hat{\boldsymbol{t}}_{B}\right)
\end{aligned}
$$

where $\tilde{\boldsymbol{f}}=\tilde{\boldsymbol{f}}_{C}=\mathbf{o}$ because $\tilde{\boldsymbol{f}}_{C}$ is the force which cannot be applied by the joint torques, and $\tilde{f}$ express an internal force (we consider this term, $\boldsymbol{\Phi} \tilde{\boldsymbol{f}}$, in the next subsection). By solving this problem, we get the following equation.

$$
\left(\begin{array}{l}
\boldsymbol{f}_{C} \\
\boldsymbol{f}_{f}
\end{array}\right)=\tilde{\boldsymbol{G}} \hat{\boldsymbol{t}}_{B}
$$

where,

$$
\begin{aligned}
\tilde{\boldsymbol{G}}= & \left(\begin{array}{c}
\boldsymbol{A}^{+}-\boldsymbol{D}_{B 0}^{T} \boldsymbol{H}_{C 0}^{T} \boldsymbol{B}_{1} \\
-\boldsymbol{B}_{1}
\end{array}\right) \\
\boldsymbol{B}_{1}= & \boldsymbol{B}_{3}^{T} \boldsymbol{B}_{4}^{+}\left\{\left(\boldsymbol{I}_{6}-\boldsymbol{A} \boldsymbol{A}^{+}\right)-\boldsymbol{B}_{3} \boldsymbol{B}_{2}^{T} \boldsymbol{J} \boldsymbol{A}^{+}\right\} \\
& +\boldsymbol{B}_{2}^{+} \boldsymbol{J} \boldsymbol{A}^{+} \\
\boldsymbol{B}_{2}= & \boldsymbol{J} \boldsymbol{A}^{+} \boldsymbol{D}_{B 0}^{T} \boldsymbol{H}_{C 0}^{T} \\
\boldsymbol{B}_{3}= & \left(\boldsymbol{I}-\boldsymbol{A} \boldsymbol{A}^{+}\right) \boldsymbol{D}_{B 0}^{T} \boldsymbol{H}_{C 0}^{T}\left(\boldsymbol{B}_{2}^{T} \boldsymbol{B}_{2}\right)^{-1} \\
\boldsymbol{B}_{4}= & \boldsymbol{B}_{3} \boldsymbol{H}_{C 0} \boldsymbol{D}_{B 0}\left(\boldsymbol{I}-\boldsymbol{A} \boldsymbol{A}^{+}\right)^{T}
\end{aligned}
$$

When we set $\boldsymbol{G}=\left(\begin{array}{ll}\boldsymbol{A} & -\boldsymbol{D}_{B 0}^{T} \boldsymbol{H}_{C 0}^{T}\end{array}\right)$, we can get $\boldsymbol{G}$ $\left[\boldsymbol{f}_{C}^{T} \boldsymbol{f}_{f}^{T}\right]^{T}=\boldsymbol{G} \tilde{\boldsymbol{G}} \hat{\boldsymbol{t}}_{B}=\hat{\boldsymbol{t}}_{B}$.

\subsection{Internal Force}

In this subsection, we consider internal forces for forming the force closure grasp. In this paper, we consider not only an internal force between the fingers but also an internal force between the finger and the environment. However, using the internal forces means that $\boldsymbol{f}_{f}$ obtained in the problem (17) don't minimize the norm of $\boldsymbol{f}_{S}$. So, in this subsection, we also consider the magnitude of the internal forces which minimizes the norm of $\boldsymbol{f}_{S}$.

First, we formulate the internal forces. The internal force satisfies

$$
\boldsymbol{G}\left(\begin{array}{l}
f_{C} \\
f_{f}
\end{array}\right)=\mathbf{o}
$$

Then, we rewrite the equation (16) as follows

$$
\left(\begin{array}{l}
\boldsymbol{f}_{C} \\
\boldsymbol{f}_{f}
\end{array}\right)=\tilde{\boldsymbol{G}} \hat{\boldsymbol{t}}_{B}+\left(\begin{array}{c}
\boldsymbol{f}_{C}^{I} \\
\boldsymbol{f}_{f}^{I}
\end{array}\right)
$$


Where, $\boldsymbol{G}\left[\left(\boldsymbol{f}_{C}^{I}\right)^{T}\left(\boldsymbol{f}_{f}^{I}\right)^{T}\right]^{T}=\mathbf{o}$, namely, $\left[\left(\boldsymbol{J} \boldsymbol{f}_{C}^{I}\right)^{T}\left(\boldsymbol{f}_{f}^{I}\right)^{T}\right]^{T}$ express the internal force.

Then, we set $\left[\left(\boldsymbol{f}_{C}^{I}\right)^{T}\left(\boldsymbol{f}_{f}^{I}\right)^{T}\right]^{T}$ as follows.

$\left(\begin{array}{c}\boldsymbol{f}_{C}^{I} \\ \boldsymbol{f}_{f}^{I}\end{array}\right)=\operatorname{diag}\left(\boldsymbol{J}_{t 1}^{-1} \boldsymbol{J}_{t 2}^{-1} \boldsymbol{I}_{3}\right) \tilde{\boldsymbol{\Lambda}} \boldsymbol{k}=\boldsymbol{\Lambda} \boldsymbol{k}$

$\tilde{\boldsymbol{\Lambda}}=\left(\begin{array}{ccc}\boldsymbol{e}_{12}+\zeta_{11} \boldsymbol{e}_{n} & \boldsymbol{e}_{10}+\zeta_{12} \boldsymbol{e}_{n} & \zeta_{13} \boldsymbol{e}_{n} \\ \boldsymbol{e}_{21}+\zeta_{21} \boldsymbol{e}_{n} & \zeta_{22} \boldsymbol{e}_{n} & \boldsymbol{e}_{20}+\zeta_{23} \boldsymbol{e}_{n} \\ \Sigma_{i=1}^{2} \zeta_{i 1} \boldsymbol{e}_{n} & \boldsymbol{e}_{10}+\Sigma_{i=1}^{2} \zeta_{i 2} \boldsymbol{e}_{n} & \boldsymbol{e}_{20}+\Sigma_{i=1}^{2} \zeta_{i 3} \boldsymbol{e}_{n}\end{array}\right)$

where $\boldsymbol{e}_{i j}$ denote a unit vector which directs from the contact point $C_{i}$ to the contact point $C_{j}, \boldsymbol{k}=\left[\begin{array}{lll}k_{1} & k_{2} & k_{3}\end{array}\right]^{T}$ denote the magnitude of the components of the internal force in the directions expressed by a column vector of $\boldsymbol{\Lambda}, \boldsymbol{e}_{n}$ denotes an arbitrary vector orthogonal to both $\boldsymbol{n}_{C 1}$ and $\boldsymbol{n}_{C 2}$, and $\boldsymbol{J}_{t i}(i=1,2)$ is a component of $\boldsymbol{J}$ as follows.

$$
\boldsymbol{J}^{T}=\left(\begin{array}{cccc}
\boldsymbol{J}_{t 1}^{T} & \boldsymbol{J}_{r 1}^{T} & \boldsymbol{O}_{3} & \boldsymbol{O}_{3} \\
\boldsymbol{O}_{3} & \boldsymbol{O}_{3} & \boldsymbol{J}_{t 2}^{T} & \boldsymbol{J}_{r 2}^{T}
\end{array}\right) \boldsymbol{H}_{C}^{T}
$$

We determine $\zeta_{i j}(i=1,2 j=1,2,3)$ in (21) by substituting (21) to (19). Then, the $\left[\left(\boldsymbol{J} \boldsymbol{f}_{C}^{I}\right)^{T}\left(\boldsymbol{f}_{f}^{I}\right)^{T}\right]^{T}$ given by $(21)$ represent internal forces. This internal forces $\left[\left(\boldsymbol{J} \boldsymbol{f}_{C}^{I}\right)^{T}\left(\boldsymbol{f}_{f}^{I}\right)^{T}\right]^{T}$ (note that $\boldsymbol{f}_{S}=\boldsymbol{J} \boldsymbol{f}_{C}$ ) includes the term(black arrows in Fig.3) given by a conventional method to determine the internal forces in the manipulation of an object by three fingers as well as the term (white arrows in Fig.3) whose direction is $\boldsymbol{e}_{n}$ and which cancels the static/dynamic frictional twist/spin moment at the contact point $C_{i}(i=1,2)$

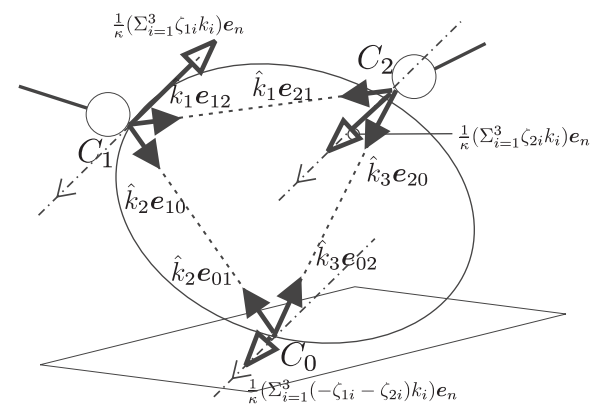

Figure 3: Internal Forces

Next, we derive a magnitude of the component of the internal force which minimizes the norm of $\boldsymbol{f}_{S}(=$ $\boldsymbol{J}_{\boldsymbol{f}_{C}}$, we consider the following nonlinear programming.

$$
\begin{aligned}
\min & h(\boldsymbol{k})=\boldsymbol{f}_{S}^{T} \boldsymbol{f}_{S}=\boldsymbol{f}_{C}^{T} \boldsymbol{J}^{T} \boldsymbol{J} \boldsymbol{f}_{C} \\
\text { subj.to. } & \left.\boldsymbol{l}(\boldsymbol{k})=\boldsymbol{V}\left(\begin{array}{c}
\boldsymbol{f}_{S} \\
\boldsymbol{f}_{f}
\end{array}\right)\right) \leq \mathbf{o}
\end{aligned}
$$

Note that $\boldsymbol{l} \leq$ o express the translational frictional constraints which approximately represent the friction cone at the contact point $C_{i}$ as the polyhedral convex cone with $L_{i}$ faces [10], where $\boldsymbol{V}=\operatorname{diag}\left[\begin{array}{lll}\boldsymbol{V}_{1} & \boldsymbol{V}_{2} & \boldsymbol{V}_{0}\end{array}\right]^{T}$,
$\boldsymbol{V}_{i}=\operatorname{col}\left[\left(a_{k}^{i}\right)^{T}\right]\left(k=1,2, \cdots, L=\Sigma_{i=1}^{3} L_{i}\right)$, and $a_{k}^{i}$ denotes a outward normal vector of the $k$ th face of the polyhedral convex cone.

In order to solve the problem (22), we introduce the following Lagrangean function $\rho(\boldsymbol{k}, \boldsymbol{\lambda})$ using Lagrange multipliers $\boldsymbol{\lambda}=\left[\lambda_{1} \lambda_{2} \cdots \lambda_{L}\right]^{T}$.

$$
\rho(\boldsymbol{k}, \boldsymbol{\lambda})=h(\boldsymbol{k})+\boldsymbol{\lambda}^{T} \boldsymbol{l}(\boldsymbol{k})
$$

Since $\boldsymbol{\Lambda}^{T} \boldsymbol{\Lambda}$ is a positive definite matrix, $\rho$ is a convex function with respect to $\boldsymbol{k}$. Then, Kuhn-Tucker optimality conditions become necessary and sufficient conditions for $\boldsymbol{k}$ to yield the global minimum. From this Kuhn-Tucker optimality conditions, we obtain ${ }^{0} \boldsymbol{k}$ as a function of $\boldsymbol{\lambda}$ (note that ${ }^{0} *$ represents the solution of *). Then, another expression of the Kuhn-Tucker optimality conditions is given by

$$
\begin{aligned}
\boldsymbol{w} & =\boldsymbol{P} \boldsymbol{\lambda}+\boldsymbol{z} \\
\boldsymbol{w}^{T} \boldsymbol{\lambda} & =0, \quad \boldsymbol{w} \geq \mathbf{0}, \quad \boldsymbol{\lambda} \geq \mathbf{0}
\end{aligned}
$$

where $\boldsymbol{w}$ denotes new variables satisfying $\boldsymbol{w}=-\boldsymbol{l}(\geq \mathbf{0})$, and $\boldsymbol{z}$ denotes the term in $-\boldsymbol{l}$ which does not related with $\boldsymbol{\lambda}$. Now, we can obtain ${ }^{0} \boldsymbol{\lambda}$ from (24) by solving linear complementarily problem [11] with respect to ${ }^{0} \boldsymbol{\lambda}$ and $\boldsymbol{w}$. However, since it is seemed to be hard to solve this problem (24), we consider to solve the problem (24) in off-line, based on the concept of contact stability proposed by Nakamura et al.(Fig.4)[12]. Since contact force and moment are influenced by an unexpected perturbation of input, we consider the following problem in place of the problem (24).

$$
\begin{aligned}
\boldsymbol{w} & =\boldsymbol{P} \boldsymbol{\lambda}+\boldsymbol{z}+\boldsymbol{V} \boldsymbol{f}_{\text {ext }} \\
\boldsymbol{w}^{T} \boldsymbol{\lambda} & =0, \quad \boldsymbol{w} \geq \mathbf{0}, \quad \boldsymbol{\lambda} \geq \mathbf{o} \\
\boldsymbol{f}_{\text {ext }} & =\left(\begin{array}{c}
\boldsymbol{f}_{\text {ext } 1} \\
\boldsymbol{f}_{\text {ext } 2} \\
\boldsymbol{f}_{\text {ext }}
\end{array}\right)=\left(\begin{array}{c}
\hat{\boldsymbol{G}}_{1} \\
\hat{\boldsymbol{G}}_{2} \\
\hat{\boldsymbol{G}}_{0}
\end{array}\right) \Delta \ddot{\boldsymbol{\phi}}_{C 0} \\
& =-\operatorname{diag}\left[\boldsymbol{J}, \boldsymbol{O}_{3}\right] \tilde{\boldsymbol{G}} \boldsymbol{M}_{B} \boldsymbol{D}_{B 0}^{-1} \boldsymbol{T}\left(\begin{array}{c}
\boldsymbol{O}_{3} \\
\boldsymbol{I}_{3}
\end{array}\right) \Delta \ddot{\boldsymbol{\phi}}_{C 0}
\end{aligned}
$$

Here, $\boldsymbol{f}_{\text {ext }}$ expresses the term related with a pertur-

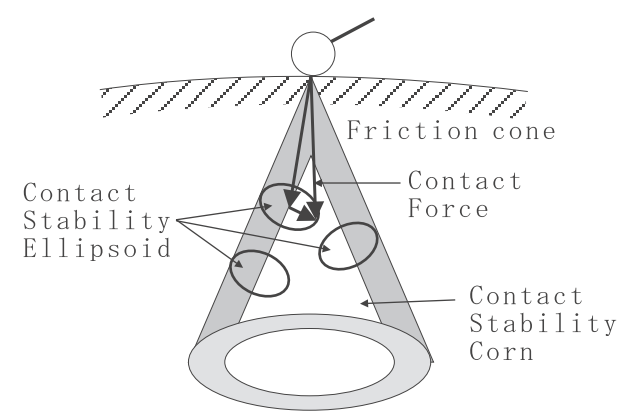

Figure 4: Contact Stability Corn and Contact Stability Ellipsoid

bation of $\ddot{\phi}_{C 0}$ (which is usually input), $\Delta \ddot{\phi}_{C 0}$. By solving this problem, we would like to get ${ }^{0} \boldsymbol{k}$ which 
makes $\boldsymbol{f}_{S i}-\boldsymbol{f}_{\text {exti }}(i=1,2)$ and $\boldsymbol{f}_{f}-\boldsymbol{f}_{\text {exto }}$ lie inside the contact stability corn shown in (Fig.4). It means the contact force and moment lie inside the friction corn even when there exist a perturbation of $\ddot{\phi}_{C 0}$. Now, by supposing a maximum magnitude of the perturbation $\left(\Delta \ddot{\phi}_{C 0}\right)$ is $\Delta \phi$, the set of $\boldsymbol{f}_{\text {ext }}$ is given by

$$
\left\{\boldsymbol{f}_{\text {exti }} \mid \boldsymbol{f}_{\text {exti }}^{T} \boldsymbol{f}_{\text {exti }} \leq \Delta \phi^{2} \hat{\boldsymbol{G}}_{i}^{T} \hat{\boldsymbol{G}}_{i}\right\}
$$

From this equation, the sets of $\boldsymbol{f}_{S i}(i=1,2)$ and $\boldsymbol{f}_{f}$ become ellipsoids (contact stability ellipsoid shown in Fig.4) with the center at $\boldsymbol{f}_{S i}-\boldsymbol{f}_{\text {exti }}(i=1,2)$ and $\boldsymbol{f}_{f}$ - $\boldsymbol{f}_{\text {ext } 0}$, respectively, and principal axes $2 \Delta \phi \sigma_{i j}(i=$ $0,1,2)$ of length, where $\sigma_{i j}(i=0,1,2)$ is the singular value of $\hat{\boldsymbol{G}}_{i}$. Then, we can solve the problem (25) and obtain ${ }^{0} \boldsymbol{\lambda}$ in off-line by setting $\boldsymbol{f}_{\text {ext }}$ in (25) as follows.

$$
\boldsymbol{f}_{\text {exti }}=\frac{\sqrt{\mu_{s i}^{2}+1}}{\mu_{s i}} \Delta \phi \sigma_{i \max } \boldsymbol{n}_{C i}
$$

where $\sigma_{\text {imax }}$ denotes the maximum value of $\sigma_{i j}$, and $\mu_{s i}(i=0,1,2)$ denotes the maximum static translational frictional coefficient at the contact point $C_{i}$.

From the obtained ${ }^{0} \boldsymbol{\lambda}$, we get ${ }^{0} \boldsymbol{k}\left(=\boldsymbol{k}_{d}\right)$.

\subsection{Controller for Pivoting}

In this subsection, we derive a controller for pivoting.

From (1) (9) (10) (11) (14), the dynamics of the system for pivoting is given by

$$
\begin{aligned}
& \boldsymbol{W} \boldsymbol{x}=\boldsymbol{b}=\operatorname{col}\left[\boldsymbol{b}_{1} \boldsymbol{b}_{2} \boldsymbol{b}_{3} \boldsymbol{b}_{4}\right] \\
& \boldsymbol{W}=\left(\begin{array}{cccc}
\boldsymbol{M}_{f} & \boldsymbol{O}_{6} & \boldsymbol{J}^{T} \boldsymbol{J} & \boldsymbol{O} \\
\boldsymbol{O}_{6} & \boldsymbol{M}_{B} & -\boldsymbol{A} & \boldsymbol{D}_{B 0}^{T} \boldsymbol{H}_{C 0}^{T} \\
\boldsymbol{J}_{C F M} & -\boldsymbol{H}_{C} \boldsymbol{D}_{B} & \boldsymbol{O} & \boldsymbol{O} \\
\boldsymbol{O} & \boldsymbol{H}_{C 0} \boldsymbol{D}_{B 0} & \boldsymbol{O} & \boldsymbol{O}_{3}
\end{array}\right) \\
& \boldsymbol{b}_{1}=\boldsymbol{\tau}-\boldsymbol{h}_{f}, \boldsymbol{b}_{2}=-\boldsymbol{h}_{B}, \boldsymbol{b}_{4}=-\boldsymbol{H}_{C 0} \dot{\boldsymbol{D}}_{B 0}\left(\begin{array}{c}
\dot{\boldsymbol{p}}_{B} \\
\boldsymbol{\omega}
\end{array}\right) \\
& \boldsymbol{b}_{3}=\left(\dot{\boldsymbol{H}}_{C} \boldsymbol{D}_{B}+\boldsymbol{H}_{C} \dot{\boldsymbol{D}}_{B}\right)\left(\begin{array}{c}
\dot{\boldsymbol{p}}_{B} \\
\boldsymbol{\omega}
\end{array}\right)-\dot{\boldsymbol{J}}_{C F M} \dot{\boldsymbol{q}}
\end{aligned}
$$

where $\boldsymbol{x}=\left[\begin{array}{llll}\ddot{\boldsymbol{q}}^{T} & \ddot{\boldsymbol{p}}_{B}^{T} & \dot{\boldsymbol{\omega}}_{B}^{T} & \boldsymbol{f}_{S}^{T} \boldsymbol{f}_{f}^{T}\end{array}\right]^{T}$.

By supposing the desired value of $\boldsymbol{x}$ is $\boldsymbol{x}_{d}=\left[\begin{array}{ll}\ddot{\boldsymbol{q}}_{d}^{T} & \ddot{\boldsymbol{p}}_{B d}^{T}\end{array}\right.$ $\left.\dot{\boldsymbol{\omega}}_{B d}^{T} \boldsymbol{f}_{S d}^{T} \boldsymbol{f}_{f d}^{T}\right]^{T}$, we can get the following controller.

$$
\begin{aligned}
\boldsymbol{\tau}= & \boldsymbol{M}_{f} \ddot{\boldsymbol{q}}_{d}+\boldsymbol{h}_{f}+\boldsymbol{J}^{T} \boldsymbol{J}_{C d} \\
\ddot{\boldsymbol{q}}_{d}= & \boldsymbol{J}_{C F M}^{+}\left\{\boldsymbol{H}_{C} \boldsymbol{D}_{B}\left(\begin{array}{c}
\ddot{\boldsymbol{p}}_{B d} \\
\dot{\boldsymbol{\omega}}_{B d}
\end{array}\right)-\dot{\boldsymbol{J}}_{C F M} \dot{\boldsymbol{q}}\right. \\
& \left.+\left(\dot{\boldsymbol{H}}_{C} \boldsymbol{D}_{B}+\boldsymbol{H}_{C} \dot{\boldsymbol{D}}_{B}\right)\left(\begin{array}{c}
\dot{\boldsymbol{p}}_{B} \\
\boldsymbol{\omega}
\end{array}\right)\right\} \\
\left(\begin{array}{c}
\ddot{\boldsymbol{p}}_{B d} \\
\dot{\boldsymbol{\omega}}_{B d}
\end{array}\right)= & \boldsymbol{D}_{B 0}^{-1} \boldsymbol{T}\left(\begin{array}{c}
\mathbf{o} \\
\boldsymbol{u}_{B}
\end{array}\right) \\
& +\left(\dot{\boldsymbol{D}}_{B 0}^{-1} \boldsymbol{T}+\boldsymbol{D}_{B 0}^{-1} \dot{\boldsymbol{T}}\right)\left(\begin{array}{c}
\mathbf{o} \\
\dot{\phi}_{C 0}
\end{array}\right)
\end{aligned}
$$

$$
\begin{aligned}
\boldsymbol{f}_{S d}= & \boldsymbol{J} \boldsymbol{f}_{C d}=\boldsymbol{J} \operatorname{diag}\left[\boldsymbol{I}_{6}, \boldsymbol{O}_{3}\right]\left\{\boldsymbol{\Lambda} \boldsymbol{u}_{I}\right. \\
& \left.+\tilde{\boldsymbol{G}}\left(\boldsymbol{M}_{B}\left(\begin{array}{c}
\ddot{\boldsymbol{p}}_{B d} \\
\dot{\boldsymbol{\omega}}_{B d}
\end{array}\right)+\boldsymbol{h}_{B}\right)\right\}
\end{aligned}
$$

where $\boldsymbol{u}_{B}$ and $\boldsymbol{u}_{I}$ are new inputs.

By applying the controller to the system (27), we get $\boldsymbol{W}\left(\boldsymbol{x}_{d}-\boldsymbol{x}\right)=\mathbf{o}$. If $\boldsymbol{W}$ is nonsingular, we can get $\boldsymbol{x}_{d}=\boldsymbol{x}$. Then, we get $\ddot{\boldsymbol{\phi}}_{C 0}=\boldsymbol{u}_{B}$ from (30) and $\boldsymbol{k}=\boldsymbol{u}_{I}$ from (21) (31).

Letting $\ddot{\boldsymbol{\phi}}_{C 0 d}$ be the desired trajectory of $\ddot{\boldsymbol{\phi}}_{C 0}, \boldsymbol{u}_{B}$ and $\boldsymbol{u}_{I}$ are given by the following servo controller

$$
\begin{gathered}
\boldsymbol{u}_{B}=\ddot{\boldsymbol{\phi}}_{C 0 d}+\boldsymbol{K}_{V}\left(\dot{\boldsymbol{\phi}}_{C 0 d}-\dot{\boldsymbol{\phi}}_{C 0}\right) \\
+\boldsymbol{K}_{P}\left(\boldsymbol{\phi}_{C 0 d}-\phi_{C 0}\right) \\
\boldsymbol{u}_{I}= \\
\boldsymbol{k}_{d}+\boldsymbol{K}_{I} \int_{0}^{t}\left(\boldsymbol{k}_{d}-\boldsymbol{k}\right) d t^{\prime}
\end{gathered}
$$

where $\boldsymbol{K}_{P}, \boldsymbol{K}_{V}$ and $\boldsymbol{K}_{I}$ are gain matrices. Then, if $\boldsymbol{W}$ is nonsingular, the object orientation error $\boldsymbol{e}_{P}=$ $\phi_{C 0 d}-\phi_{C 0}$ and the force error $\boldsymbol{e}_{f}=\boldsymbol{k}_{d}-\boldsymbol{k}$ satisfy the following equations

$$
\begin{aligned}
\ddot{e}_{P}+\boldsymbol{K}_{V} \dot{\boldsymbol{e}}_{P}+\boldsymbol{K}_{P} \boldsymbol{e}_{P}=\mathbf{o} \\
\dot{\boldsymbol{e}}_{f}+\boldsymbol{K}_{I} \boldsymbol{e}_{f}=\mathbf{o}
\end{aligned}
$$

Thus, with appropriate $\boldsymbol{K}_{P}, \boldsymbol{K}_{V}$ and $\boldsymbol{K}_{I}$, the actual object orientation $\ddot{\phi}_{C 0}$ and the component of the internal forces $\boldsymbol{k}$ will converge to the desired trajectories asymptotically.

\section{Simulation}

We show simulation results in this section to verify the validity of our approach. Suppose that the object is a regular prism whose bottom is a regular pentagon whose side is $50[\mathrm{~mm}]$ length, whose height is $100[\mathrm{~mm}]$ length, and whose weight is $500[\mathrm{~g}]$. Each finger is a 3 D.O.F. parallel link robot (Fig.1), and each fingertip is a spherical soft fingertip with $15[\mathrm{~mm}]$ radius. The orientation of the object is expressed by $\operatorname{roll} \phi, \operatorname{pitch} \theta$, yaw $\psi$.

We set each dynamic rotational frictional coefficient $\left(\mu_{i}\right)$ is 2.0 , and each maximum static rotational frictional coefficient $\left(\tilde{\mu}_{i}\right)$ is 2.2 . We also set the $\max -$ imum static translational frictional coefficients $\left(\mu_{s i}\right)$ at the contact point $C_{1}, C_{2}, C_{0}$ is $1.0,1.0,0.8$, respectively. We consider the desired trajectory as follows; First, tilting (raising) the object around the vertex $P\left(\right.$ Fig.5(a)) for the interval from $t_{0}(=0.0[\mathrm{sec}])$ to $t_{1}(=0.5[\mathrm{sec}])$, Then, rotating the object around the vertex $P$ (Fig.5(b)) for the interval from $t_{1}(=0.5[\mathrm{sec}])$ to $t_{2}(=1$. [sec]), Finally, lowering the object around the vertex $P(\mathbf{F i g . 5}(\mathrm{c}))$ for the interval from $t_{2}(=1$. $[\mathrm{sec}])$ to $t_{3}(=1.5[\mathrm{sec}])$.

We set the maximum magnitude of the perturbation of $\ddot{\phi}_{C 0}, \Delta \phi_{i}$ is a proportional value to $\left|\ddot{\phi}_{C 0}\right|(10 \%)$, and represent the friction cone at each contact point $C_{i}$ as the polyhedral convex cone with 5 faces. 
Servo gain are set to $\boldsymbol{K}_{P}=[5000,5000,5000]$, $\boldsymbol{K}_{V}=[200,200,200]$, and $\boldsymbol{K}_{I}=[10,10,10]$, and sampling time are set to $2.0[\mathrm{msec}]$.

First, we calculate $\boldsymbol{k}_{d}$ in off-line. Then, we simulate the pivoting. The result is shown in Fig.6. Fig.6(a) shows the actual object orientation and the desired one, and Fig.6(b) shows the differences of inner product between each contact normal and unit vector expressing the direction of each contact force. The value 0 of the differences means contact force lie in the borderline of the friction corn, and dot line express max value of the differences. From the Fig.6(a), we can see the actual object orientation converge to the desired orientation(note that we can not see the difference between actual trajectories and desired trajectories). From the Fig.6(b), we can see the contact forces satisfy the frictional constraints and the contact forces of finger $i$ lie in the region close to the border of the frictional constraints.

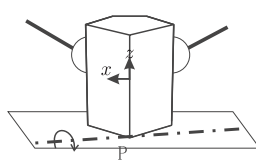

(a)

Figure 5: Transition of State
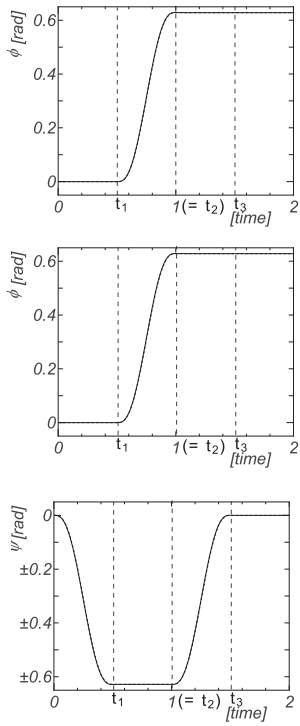

Figure 6: Simulation Result of Pivoting
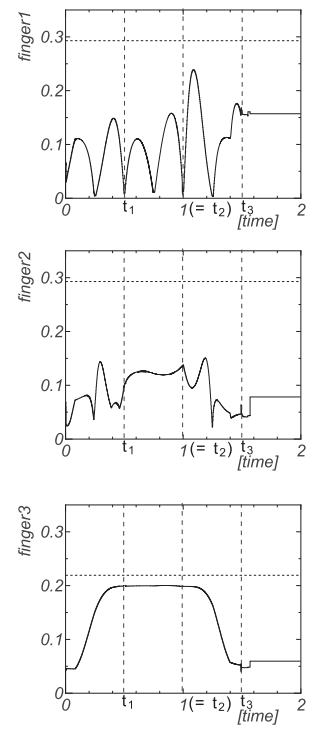

\section{Conclusions}

In this paper, we have proposed a dynamic control method of soft-finger hands for pivoting an object in contact with the environment. The characteristics of the pivoting are that we can use a reaction force from the environment. By using this reaction force, we can expect the magnitude of the forces applied to the object by the fingers is smaller than the conventional manipulation of the object by only fingers. In this paper, taking this characteristics of the reaction force into consideration, we propose a dynamic control method for pivoting. To verify our approach, we have also presented the simulation result.

\section{References}

[1] Z. Li, P. Hsu and S. Sastry, "Grasping and Coordinated Manipulation by a Multifingered Robot Hand," The International Journal of Robotics Research, Vol. 8, No.4, pp. 33-50, 1989.

[2] A.B.A. Cole, J.E.Hauser and S.S.Sastry, "Kinematics and Control of Multifingered Hands with Rolling Contact," IEEE Trans. on Automatic Control, Vol.34, No.4, pp. 398-404, 1989.

[3] Y. Yokokouji, M. Sakamoto and T. Yoshikawa, "Vision-Aided Object Manipulation by a Multifingered Hand with Soft Fingertips," Proc. IEEE International Conference on Robotics and Automation, pp. 3201-3208, 1999.

[4] A. Bicchi, "On the Closure Properties of Robotic Grasping," The International Journal of Robotics Research, Vol.14, No.4, pp. 319-334, 1995.

[5] Y. Aiyama, M. Inaba and H. Inoue, "Pivoting: A New Method of Graspless Manipulation of Object by Robot Fingers," Proc. IEEE/RSJ International Conference on Intelligent Robots and Systems, pp. 136$143,1993$.

[6] T. Yoshikawa and X. Zheng, "Coordinated Dynamic Hybrid Position/Force Control for Multiple Robot Manipulators Handling One Constrained Object," The International Journal of Robotics Research, Vol.12, No.3, pp. 219-230, 1993.

[7] L. Han, Y. S. Guan, Z. X. Li, Q. Shi and J. C. Trinkle, "Dexterous Manipulation with Rolling Contacts," Proc. IEEE International Conference on Robotics and Automation, pp. 992-997, 1997.

[8] J. C. Trinkle and R. P. Paul, "Planning for Dexterous Manipulation with Sliding Contacts," The International Journal of Robotics Research, Vol.9, No.3, pp. 24-48, 1990.

[9] J. C. Trinkle, R. C. Ram, A. O. Farahat and P. F. Stiller, "Dexterous Manipulation Planning and Execution of Enveloped Slippery Workpiece," Proc. IEEE International Conference on Robotics and Automation, pp. 442-448, 1993.

[10] J. Kerr and B. Roth, "Analysis of Multifingered Hands," International Journal of Robotics Research, Vol.4, No.4, pp. 3-17, 1986.

[11] C. E. Lemke, "Bimatrix Equilibrium Points and Mathematical Programming,"Man. Sci., Vol.11, pp. 681-689, 1965 .

[12] Y. Nakamura, K. Nagai and Y. Yoshikawa, "Dynamics and Stability in Coordination of Multiple Robotic Mechanisms," International Journal of Robotics Reserach, Vol.8, No.2, pp. 44-61, 1989. 